\title{
Amyloid- $\beta$-induced matrix metalloproteinase-9 secretion is associated with retinal pigment epithelial barrier disruption
}

\author{
LINING CAO, HAO WANG and FANG WANG \\ Department of Ophthalmology, Shanghai Tenth People's Hospital affiliated with \\ Tongji University, School of Medicine, Shanghai, P.R. China
}

Received December 22, 2012; Accepted February 12, 2013

DOI: $10.3892 / \mathrm{ijmm} .2013 .1310$

\begin{abstract}
Local and chronic inflammation induced by amyloid- $\beta$ (A $\beta)$ plays a central role in the development of age-related macular degeneration. The retina is an immuneprivileged site due to local tissue barrier. Yet, the manner by which immune cells pass through this barrier and accumulate in the retina remains unclear. Matrix metalloproteinases (MMPs) induce barrier disruption via proteolysis of epithelial tight junction (TJ) proteins. We hypothesized that $A \beta$-induced MMP secretion causes disruption of epithelial barrier integrity. To test this hypothesis, human adult retinal pigment epithelial (haRPE) cells were exposed to A $\beta$, and the expression of MMP-2 and MMP-9 was detected using gelatin zymography. To demonstrate the key role of MMPs in modulating epithelial barrier structure, the MMP agonist 4-aminophenylmercuric acetate (APMA), an MMP inhibitor (GM6001) and siRNA against MMP-9 were employed for comparison. We found that MMP-9, secreted by A $\beta$ - or APMA-stimulated cells, mediated low transepithelial electrical resistance (TER) and high transepithelial permeability by disrupting TJ proteins. However, these alterations were reduced by the MMP inhibitor GM6001 or by silencing of the MMP-9 gene. Our findings suggest that the degradation of TJ proteins such as zonula occludens-1, occludin and F-actin by MMP-9 secreted by A $\beta$-stimulated cells constitutes an important mechanism in the breakdown of the barrier which contributes to chronic inflammation in the retina of agerelated macular degeneration.
\end{abstract}

\section{Introduction}

Age-related macular degeneration (AMD) is a leading cause of irreversible visual loss, affecting $5-10 \%$ of the population

Correspondence to: Professor Fang Wang, Department of Ophthalmology, Shanghai Tenth People's Hospital, No. 301 Middle Yanchang Road, Shanghai 200072, P.R. China

E-mail: 18917683335@163.com

Key words: age-related macular degeneration, barrier integrity, tight junction, matrix metalloproteinase-9, GM6001, 4-aminophenylmercuric acetate, RNA interference over 60 years of age in Western countries (1). Drusen represent the earliest sign of AMD and are also high risk factors for the development of AMD complications (2). Amyloid- $\beta$ (A $\beta)$, a known constituent of drusen, is a contributor to the development of AMD (3-5). Accumulated evidence has confirmed that the $\mathrm{A} \beta$-mediated inflammatory response is associated with the pathology of AMD (6-8).

The retina is an immune-privileged site due to local tissue barrier (9). Yet, the manner by which immune cells pass through this barrier and accumulate in the retina remains unclear. Retinal pigment epithelial (RPE) cells in the retina form a monolayer between photoreceptors and choroidal vessels which is a barrier to maintain the structural and functional integrity of the retina. The blood-retinal barrier (BRB) is composed of the inner BRB (iBRB) consisting of retinal microvascular endothelial cells and the outer BRB (oBRB) consisting of RPE cells. Alterations in the BRB play a crucial role in the development of $\operatorname{AMD}(10,11)$. The formation of the oBRB by RPE cells is dependent on the structure of tight junction (TJ) proteins, including the transmembrane protein occludin, claudins and zonula occludens-1 (ZO-1). Zonula occludens interact with the transmembrane proteins at the cytoplasmic face of the cell membrane and serve to anchor them to the actin cytoskeleton (actin filaments, F-actin). Abnormal expression or localization of occludin, F-actin, and ZO-1 in RPE cells contributes to barrier dysfunction $(12,13)$. Matrix metalloproteinase (MMP)-2 and 9 were reported to cause barrier disruption by abnormal degradation of $\mathrm{TJ}$ proteins $(14,15)$.

A recent study reported that $A \beta$ caused morphological alterations and barrier dysfunction in ARPE-19, a spontaneously transformed cell line (16). Yet, the underlying mechanism is poorly understood. Therefore, we aimed to ascertain whether A $\beta$-induced RPE barrier disruption is mediated by MMPs. To note, the immortalized human retinal pigment epithelial cell line ARPE-19 may not exhibit important properties similar to the native tissue; the cell line is not ideal for studying outer retinal barrier function because of its abnormal junctional complexes and the lower transepithelial electrical resistance (TER) (17). Therefore, isolated human adult RPE cells were used, for the first time to the best of our knowledge, to explore the effect of $A \beta$ on epithelial barrier integrity.

Although $A \beta$-induced disruption of the RPE structure and breakdown of the barrier function were observed in our 
previous study, little is known concerning the mechanism that contributes to $A \beta$-induced RPE alterations. Since $A \beta$-induced chronic inflammation is strongly linked to the pathology of $\operatorname{AMD}(7,8)$, inflammatory cytokines are speculated to be one of the primary causative events that contribute to $\mathrm{A} \beta$-induced barrier dysfunction. In addition to causing inflammation, some pro-inflammatory factors such as IL- 6 can alter tissue microenvironments by the modulation of epithelial TJ proteins (18). It is worth mentioning that MMPs have been reported to act broadly in inflammation to regulate barrier function (19-21). Microarray analysis of the gene expression profile of ARPE-19 cells in response to A $\beta$ stimulation suggests that IL- 8 is significantly upregulated by $A \beta$ (8). Higher intraocular concentrations of IL-6, IL-8 and MMP-9 have been measured in AMD patients and are significantly associated with the severity of the disease $(22,23)$. Therefore, in the present study expression levels of IL-6, IL-8, MMP-2 and MMP-9 were analyzed in the A $\beta$-stimulated RPE cells. An increase in MMP activity has been causally linked to epithelial barrier disruption and severe symptoms of inflammatory diseases (24-26). To demonstrate the key role of MMPs in modulating epithelial barrier structure and function, general proteinase agonist 4-aminophenylmercuric acetate (APMA) was used to induce MMP activation. For inhibitory studies, MMP-2 and MMP-9 activities were inhibited by RNA interference strategy or by the MMP inhibitor GM6001.

\section{Materials and methods}

$A \beta_{1-42}$ oligomerization and dot blot assay. Lyophilized $\mathrm{A} \beta_{1-42}$ peptide (Sigma-Aldrich) was dissolved to a concentration of $1.5 \mathrm{M}$ in hexafluoroisopropanol (HFIP) on ice and aliquoted at $-20^{\circ} \mathrm{C}$. A $\beta$ monomers were spin-vacuumed just prior to the experiment, diluted to $250 \mu \mathrm{M}$ in HFIP solution, and maintained at room temperature (RT) for 3 days to synthesize $A \beta$ oligomers. To note, In the present study, references to $A \beta$ or $\mathrm{OA} \beta$ are to the oligomeric form of $\mathrm{A} \beta_{1-42}$, unless otherwise stated.

Several lines of evidence have demonstrated that soluble oligomers of $A \beta$ may be better correlated with the severity of the disease than are monomers or insoluble amyloid fibrils $(3,27)$. Therefore, a dot blot assay was performed to determine the oligomeric form of $A \beta_{1-42}$. Five microliters of oligomeric $A \beta_{1-42}$ solution and monomers of $A \beta_{1-42}$ were spotted onto a nitrocellulose membrane. The membrane was then blocked with 5\% dry milk in TTBS $(50 \mathrm{mM}$ Tris, $0.05 \%$ Tween-20) for $1 \mathrm{~h}$ at RT, and washed three times before being incubated for $1 \mathrm{~h}$ at RT with the A11 (27), an antioligomer antibody (1:1,000; Invitrogen, Carlsbad, CA, USA). The membrane was then washed and incubated for $1 \mathrm{~h}$ at RT with a HRP-conjugated secondary antibody (1:1,000 goat anti-rabbit IgG; Santa Cruz Biotechnology, Inc. Santa Cruz, CA, USA). The blots were washed three times in TTBS and incubated with chemiluminescent reagent, and finally exposed to ImageQuant LAS 4,000.

Ethics. Informed consent for tissue donation was obtained from the relatives of the donors, and the protocol of the study was approved by the local ethics committee and adhered to the tenets of the Declaration of Helsinki for experiments involving human tissue.

Isolation of human RPE cells. Five human donor eyes were obtained from the eye bank of the Eye and ENT Hospital of Fudan University, Shanghai, China. The donors ranged in age from 30 to 40 years. None of the donors had a history of eye disease. In brief, whole eyes were cleansed in $0.9 \% \mathrm{NaCl}$ solution, immersed in 5\% polyvinylpyrrolidone iodine and rinsed again in $\mathrm{NaCl}$ solution. Then the anterior segment from each eye was removed. The neural retina was peeled away from the RPE-choroid-sclera. The eyecup was rinsed with $\mathrm{Ca}^{2+}$ and $\mathrm{Mg}^{2+}$-free Hank's balanced salt solution and treated with $0.25 \%$ trypsin for $1 \mathrm{~h}$ at $37^{\circ} \mathrm{C}$. The trypsin was aspirated and replaced with DMEM/F12 (HyClone) supplemented with $20 \%$ fetal calf serum (FCS).

Cell viability assay. To measure cytotoxicity, haRPE cells seeded in a 96-well plate were treated with $0.3 \mu \mathrm{M}$ of OA $\beta$ (Sigma-Aldrich, China), $20 \mu \mathrm{g} / \mathrm{ml}$ of GM6001 (Calbiochem, Germany), $100 \mu \mathrm{M}$ of APMA (Sigma-Aldrich) for $24 \mathrm{~h}$. Cell viability was measured by the addition of 3-(4,5-dimethylthiazol-2-yl)-5-(3-carboxymethoxyphenol)2-(4-sulphophenyl)-2H-tetrazolium (MTS; Promega, USA) for $3 \mathrm{~h}$ at $37^{\circ} \mathrm{C}$. The optical density was measured spectrophotometrically at $490 \mathrm{~nm}$ on a microplate reader.

Enzyme-linked immunosorbent assay. The protein concentrations of IL- 8 and IL- 6 were determined using ELISA kits (R\&D Systems, Minneapolis, MN, USA) and were calculated based on standard curves. The optical density was measured at $490 \mathrm{~nm}$ on a microplate reader.

Transfection of haRPE cells with small interfering RNA (siRNA). Human MMP-9-specific siRNAs were purchased from Santa Cruz Biotechnology, Inc. For RNA interference experiments, cells were plated at a density of $4 \times 10^{5}$ cells/well in a 6-well plate. After $24 \mathrm{~h}$, cells were transfected with siRNA using Lipofectamine 2000 (Invitrogen), according to the manufacturer's instructions.

Gelatin zymography. To examine whether A $\beta$ induces MMP secretion, gelatin zymography was carried out as previous described (19). Briefly, the supernatant was collected after treatment and subjected to SDS-PAGE in $10 \%$ polyacrylamide gels with $1 \mathrm{mg} / \mathrm{ml}$ gelatin. After electrophoresis, gels were incubated in $2.5 \%$ Triton X-100 $\left(1 \mathrm{~h}, 37^{\circ} \mathrm{C}\right)$ followed by overnight incubation in $50 \mathrm{mM}$ Tris- $\mathrm{HCl}(\mathrm{pH} 7.8), 5 \mathrm{mM}$ $\mathrm{CaCl}_{2}, 0.02 \% \mathrm{NaN}_{3}, 0.02 \%$ Brij gels, and were stained with 2.5\% Coomassie Blue R-250 (Bio-Rad) for 45 min followed by destaining in deionized water with $10 \%$ acetic acid and $20 \%$ methanol. Gels were scanned and the density analyses of the bands were performed using Photoshop CS4.0.

Cell morphology and immunofluorescence staining. The barrier function of RPE cells is considered highly dependent on the integrity of TJ proteins and F-actin. To investigate the effects of OA $\beta$, RPE cell morphology, structures of F-actin, and location of TJ proteins were examined by light microscopy and confocal microscopy, respectively. The haRPE cells 

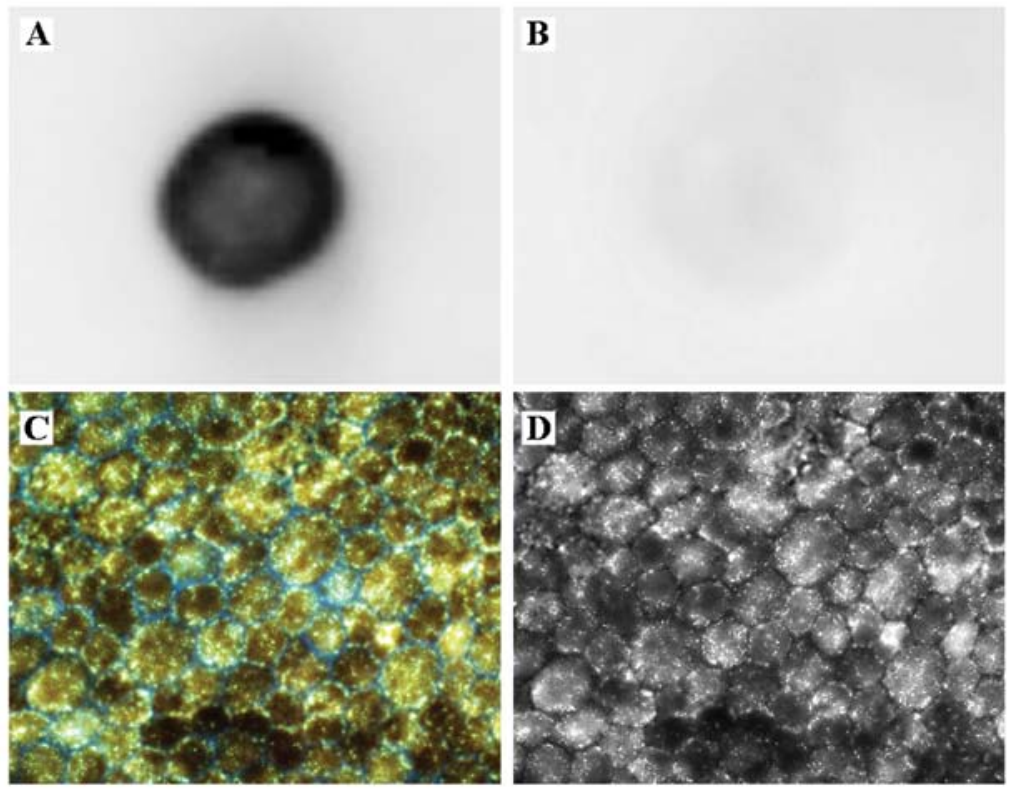

Figure 1. Confirmation of oligomeric form of $\mathrm{A} \beta_{1-42}$ and images of primary haRPE cells. Lyophilized $\mathrm{A} \beta$ peptide was dissolved, and the fresh aliquots were incubated at room temperature for 12 or $72 \mathrm{~h}$ respectively. (A) Dot-blot assay using $5 \mu 1$ of oligomeric $\mathrm{A} \beta_{1-42}$ probed with A11 antibody. Positive staining was detected in the aliquot incubated for $72 \mathrm{~h}$ to form oligomers. (B) Dot-blot assay using $5 \mu \mathrm{l}$ of A $\beta_{1-42}$ monomer probed with A11 antibody. Negative staining was shown in the monomer of $A \beta$ samples, which was incubated for $12 \mathrm{~h}$ only. (C) Fluorescence microscopic image of haRPE cells. (D) 'a' channel image in LAB color mode of haRPE cells. The primary RPE cells were hexagonal in shape and densely pigmented throughout. Magnification, $\mathrm{x} 400$.

were treated with $\mathrm{A} \beta(0.3 \mu \mathrm{M})$ for $72 \mathrm{~h}$. We hypothesized that A $\beta$-induced MMP-9 secretion causes disruption of epithelial barrier integrity. To test this hypothesis, cells were treated with APMA $(100 \mu \mathrm{M})$ for $6 \mathrm{~h}$ to induce MMP activation. For inhibitory studies, MMP-9 activity was inhibited by MMP-9 siRNA or by the MMP inhibitor GM6001. Then cells were fixed in $4 \%$ paraformaldehyde (PFA) for $30 \mathrm{~min}$ and blocked with $1 \%$ BSA in TBS for $1 \mathrm{~h}$, then incubated with rabbit anti-occludin antibody or mouse anti-ZO-1 antibody (Abcam, Hong Kong) for $1 \mathrm{~h}$. After washing, they were incubated with AlexaFluor 488-conjugated secondary antibody (1:500; Invitrogen) and DAPI $(1: 1,000)$ for $1 \mathrm{~h}$. Changes in F-actin structures were detected by FITC-labeled phalloidin (1:200; Beyotime, China). Then slides were viewed using a Leica SP5 scanning confocal microscope.

Measurement of TER. RPE cell cultures were grown on a microporous filter to form monolayers. TER was measured using Millicell-ERS (Millicell; Millipore, Bedford, MA, USA) and calculated by subtracting the value of a blank filter without cells from the experimental value. Final resistance-area products $\left(\Omega \times \mathrm{cm}^{2}\right)$ were obtained by multiplying the TER by the effective growth area. Fifteen days after TER stabilization, the monolayers were incubated with or without one of the stimuli: $\mathrm{A} \beta(0.3 \mu \mathrm{M})$, APMA $(100 \mu \mathrm{M})$, GM6001 $(20 \mu \mathrm{g} / \mathrm{ml})$. To demonstrate the key role of MMP-9 in regulating barrier function, MMP-9 silenced haRPE cells were exposed to $\mathrm{A} \beta(0.3 \mu \mathrm{M})$. Measurements were repeated at least four times for each filter, and each experiment was repeated at least four times.

Permeability assay. The permeability assays were performed by measuring the passive permeation of FITC-dextran $(4 \mathrm{kDa}$;
Sigma-Aldrich) across confluent cells grown on filters. Fifteen days later, the monolayers were treated as previously described. FITC-dextran $(500 \mathrm{mg} / \mathrm{ml})$ was added to the upper chamber on day 21 . Samples $(100 \mu \mathrm{l})$ were taken from the upper and lower chamber $24 \mathrm{~h}$ after addition of FITC-dextran. The concentration of FITC-dextran in these samples was quantified by a microplate reader (ex1800; Biotek, Winooski, VT, USA). Each experiment was repeated four times.

Statistical analysis. Data were analyzed with the software SPSS 11.5. Results were expressed as means \pm SEM. Values were processed for statistical analysis (unpaired t-test or by ANOVA), and differences were considered statistically significant at $\mathrm{P}<0.01$ and $\mathrm{P}<0.05$.

\section{Results}

Characterization of $A \beta_{1-42}$ oligomers and morphology of haRPE cells. Samples were examined after incubation for 12 or $72 \mathrm{~h}$. Dot blot assay with the anti-oligomeric specific antibody (A11) further confirmed the presence of oligomeric structures as it reacted positively with our OA $\beta$ sample (Fig. 1A). Furthermore, the A11 antibody did not react against a monomer (Fig. 1B) confirming the specificity of the antibody to the oligomeric form.

Structural integrity is the basis of good function. To confirm that the isolated haRPE cells displayed classical morphology (uniform hexagonal arrays of cells), confluence and uniform pigmentation as in native tissue, the morphology of the cells was evaluated using fluorescence microscopy (Fig. 1C). Images captured by fluorescence microscopy were analyzed using image editing software (Photoshop CS4) in the 'a' channel of LAB color mode. Apparent regular hexagonal morphology of 

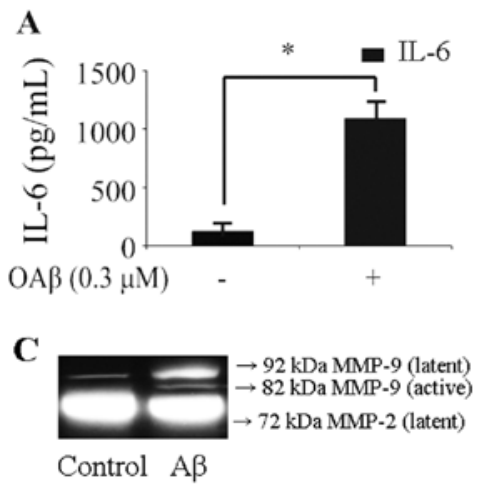
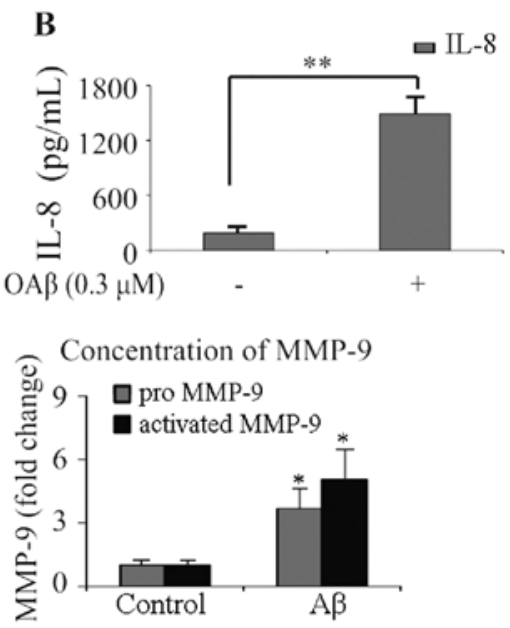

Figure 2. Expression of IL-6, IL-8, MMP-2 and MMP-9 in haRPE cells. (A) ELISA analysis of the protein level of proinflammatory cytokine IL-6. (B) ELISA analysis of the protein level of proinflammatory cytokine IL-8. (C) Detection of MMP-2 and MMP-9 secretion using gelatin zymography. The result showed that concentrations of (A) IL-6 , (B) IL-8 and (C) MMP-9 were significantly increased in RPE cells stimulated with OA $\beta(0.3 \mu \mathrm{M})$ for $24 \mathrm{~h}$ when compared with that of the control group $\left({ }^{* *} \mathrm{P}<0.01{ }^{*} \mathrm{P}<0.05\right)$.

A

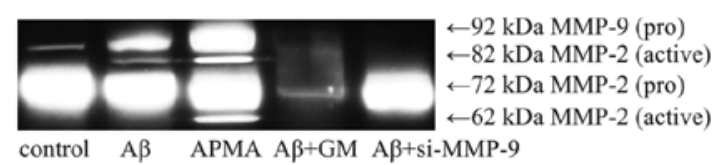

B
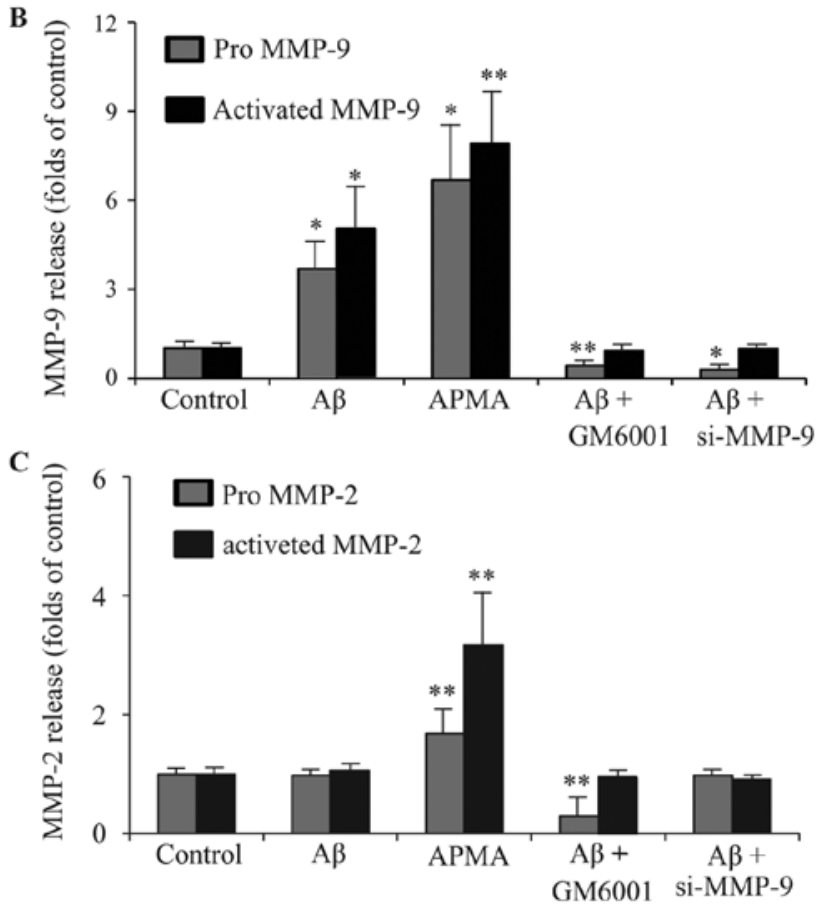

Figure 3. Influence of APMA, GM6001 and MMP-9 siRNA on MMP expression in RPE cells. MMP agonist APMA $(100 \mu \mathrm{M})$ and MMP inhibitor GM6001 $(20 \mu \mathrm{g} / \mathrm{ml})$ were used to stimulate haRPE cells for $6 \mathrm{~h}$. The MMP-9 gene was silenced by transfection of haRPE cells with $40 \mathrm{nM}$ of MMP-9 siRNA for $24 \mathrm{~h}$. (A) Secreted levels of MMP-2 and MMP-9 were detected by gelatin zymography. (B) Secreted level of MMP-9 was quantified by calculating the density of each band. (C) Secreted level of MMP-2 was quantified by calculating the density of each band. Secretion of MMPs from control cells was characterized by low levels of pro-MMP-9, absent levels of activeMMP-9, considerable output of pro-MMP-2, and absence of active-MMP-2 Treatment with APMA significantly increased the secretion of pro-MMP-2 and pro-MMP-9, as well as the activated form of MMP-2 and MMP-9. Treatment with GM6001 or silencing of MMP-9 reduced the increase in pro-MMP-9 and active-MMP-9 resulting from $\mathrm{A} \beta$ treatment. the cells was observed (Fig. 1D). This result confirmed that the isolated haRPE cells exhibited heavy pigmentation (Fig. 1C) and hexagonal epithelial morphology (Fig. 1C and D) similar to these features in native tissue (17).

MTS assay of cytotoxicity. Effects of GM6001 $(20 \mu \mathrm{g} / \mathrm{ml})$ and APMA $(100 \mu \mathrm{M})$ on cell viability were demonstrated at $24 \mathrm{~h}$. None of the measurements showed significant cytotoxicity for the treatments at the concentrations used in the present study (data not shown).

Expression of MMP-9 and proinflammatory cytokines in $A \beta$-stimulated haRPE cells. Since the inflammatory response is speculated to be one of the primary causative events that contributes to OA $\beta$-induced RPE degeneration, haRPE cells were treated with $0.3 \mu \mathrm{M}$ of OA $\beta$ for $24 \mathrm{~h}$, and the conditioned medium was collected. Expression of IL-6 and IL-8 was analyzed using ELISA (Fig. 2A and B), and the secreted levels of MMP-2 and MMP-9 were detected by gelatin zymography (Fig. 2C). The protein levels of IL-6 and IL-8 in the culture media of unstimulated RPE cells were 123.6 \pm 62.5 and $190.9 \pm 68.2 \mathrm{pg} / \mathrm{ml}$, respectively. In OA $\beta$-stimulated cells, the protein level of IL-6 increased to $1086.2 \pm 153.3 \mathrm{pg} /$ $\mathrm{ml}$ (Fig. 2A), and the concentration of IL-8 increased to $1493.2 \pm 182.3 \mathrm{pg} / \mathrm{ml}$ (Fig. 2B). MMP release from the control cells was characterized by low levels of pro-MMP-9, absent levels of active-MMP-9, considerable output of pro-MMP-2, and absence of active-MMP-2. Exposure to OA $\beta$ significantly increased secreted levels of pro-MMP-9 (3.8 \pm 0.8 -fold, $\mathrm{P}<0.05)$ and active-MMP-9 $(5.1 \pm 1.2$-fold, $\mathrm{P}<0.05)$, while treatment with $\mathrm{OA} \beta$ did not induce any change in the secreted levels of pro-MMP-2. Active foms of MMP-2 were not observed in the OA $\beta$-stimulated cells. These results suggest that OA $\beta$ induces haRPE cells to secrete higher concentrations of IL-6 and IL-8, which are critical chemoattractants responsible for immune-cell recruitment. In addition, the results revealed that OA $\beta$ induced the release of pro-MMP-9 and active-MMP-9 from haRPE cells. 


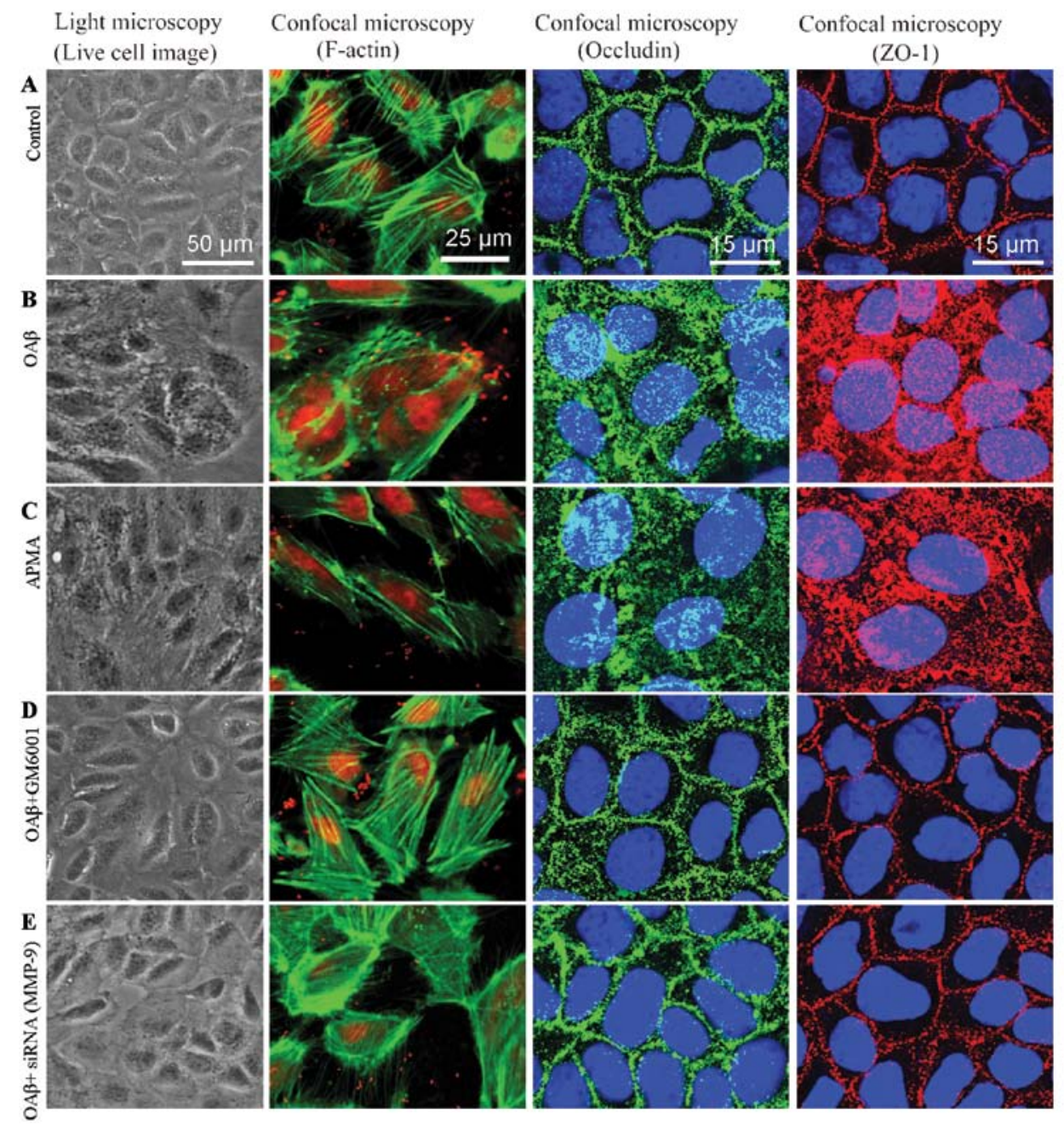

Figure 4. Effects of A $\beta$, APMA, GM6001 and MMP-9 siRNA on the morphology and structures of TJ proteins in RPE cells. RPE cells were incubated with varying stimuli for $48 \mathrm{~h}$ as follows: (A) DMEM/F12 medium, (B) $0.3 \mu \mathrm{M} \mathrm{OA \beta}$, (C) $100 \mu \mathrm{M}$ APMA or (D) $20 \mu \mathrm{g} / \mathrm{ml} \mathrm{GM} 6001$ for $6 \mathrm{~h}$ before addition of OA $\beta$ or (E) exposure of MMP-9-silenced haRPE cells to OA $\beta$. Light microcopy showed that cells treated with (B) OA $\beta$ or (C) APMA displayed irregular morphology when compared with the (A) control, while pretreatment with (D) GM6001 or (E) transfection of haRPE cells with MMP-9 siRNA reversed the effects induced by A $\beta$. Actin staining of the haRPE cells with phalloidin was used to define the altered area of the F-actin cytoskeleton. Loss of phalloidin staining was observed in (B) OA $\beta$ and (C) APMA-treated cells. In cells pretreated with (D) GM6001 or in (E) MMP-9-silenced haRPE cells, no obvious difference in actin staining was observed when compared with the (A) control. Immunostaining of occludin and ZO-1 showed that the localization of occludin and ZO-1 perfectly matched the typical cobblestone-like morphology of RPE cells in the (A) control, whereas immunostaining showed not only disorganization of occludin and ZO-1, but also disappearance of typical cobblestone-like morphology in (B) OA $\beta$ or (C) APMA-treated cells. Inhibition of MMP-9 activity by GM6001 or by MMP-9 siRNA partially attenuated the alterations of occludin and ZO-1 induced by OA $\beta$.

Influence of APMA, GM6001 and MMP-9 siRNA on MMP release from RPE cells. A $\beta$ has been shown to affect the TJs of ARPE-19 cells (16). Uncontrolled increase in MMP-9 activity has been causally linked to epithelial barrier disruption as the result of abnormal degradation of TJ proteins (21). Increased MMP-9 activity on the ocular surface disrupted corneal epithelial barrier function due to proteolytic cleavage of occludin (26). We hypothesized that A $\beta$-induced RPE disruption is mediated by activation of MMP-9. Therefore, MMP agonist APMA $(100 \mu \mathrm{M})$ and MMP inhibitor GM6001 $(20 \mu \mathrm{g} /$ $\mathrm{ml})$ were used to stimulate haRPE cells for $6 \mathrm{~h}$. The MMP-9 gene was silenced by transfection of haRPE cells with $40 \mathrm{nM}$ of MMP-9 siRNA for $24 \mathrm{~h}$. Release of MMP-9 and MMP-2 from haRPE cells was then detected by gelatin zymography (Fig. 3A), and the secreted levels of MMP-2 and MMP-9 were quantified by densitometry (Fig. 3B and C). Secretion of MMPs from control cells was characterized by low levels of pro-MMP-9, absent levels of active-MMP-9, considerable output of pro-MMP-2 and absence of activeMMP-2. Treatment with APMA significantly increased secretion of pro-MMP-2 and pro-MMP-9, as well as the activated form of MMP-2 and MMP-9. Treatment with GM6001 or silencing of MMP-9 reduced the increase in pro-MMP-9 and active-MMP-9 resulting from $\mathrm{A} \beta$ treatment.

$A \beta$-induced disruption of barrier integrity is mediated by MMP-9 activity. Recent studies involving several CNS diseases suggest that MMP-9 is involved in the permeability of the blood-brain barrier by disrupting junction complexes $(15,20)$. Giebel et al (28) found that RPE cells treated with purified MMP-9 displayed alterations in tight junction function as shown by decreased TER. Therefore, we hypothesized that OA $\beta$-induced RPE barrier disruption is mediated by activation of MMP-9. To test this 
A

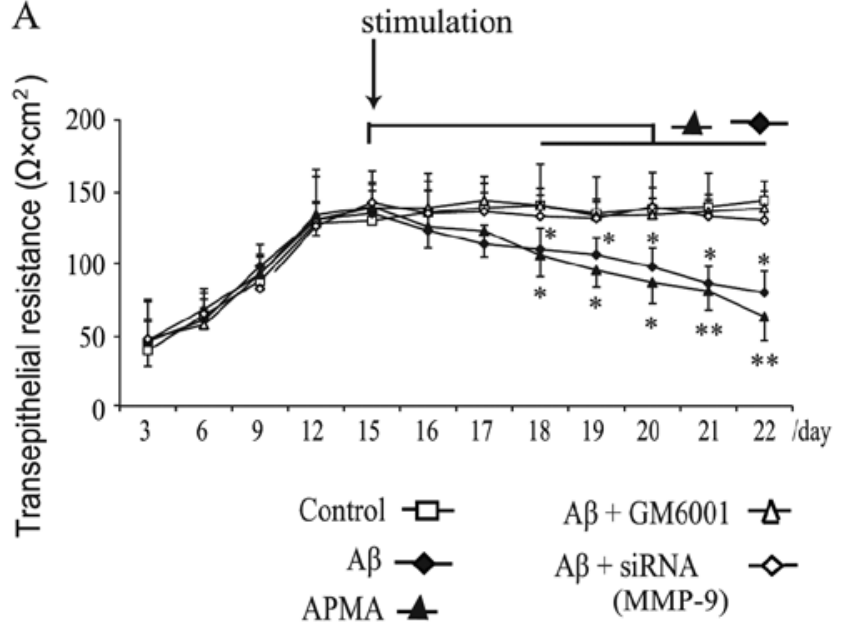

B

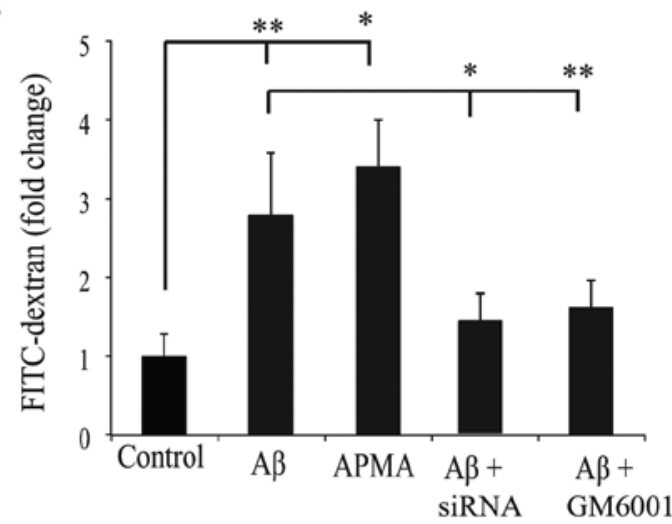

Figure 5. Effect of A $\beta$, APMA, GM6001 and MMP-9 siRNA on the barrier integrity of the RPE cell monolayer. (A) Measurement of TER in haRPE cells. Monolayers were cultured for 15 days for TER stabilization. Incubation of RPE cells with A $\beta$ or APMA induced a significant decrease in TER at day 18 and thereafter. Inhibition of MMP-9 activity by GM6001 or by MMP-9 siRNA prevented a reduction in TER induced by OA $\beta$. (B) Analysis of transepithelial permeability by measuring the passive permeation of FITC-dextran. Stimulation of RPE cells with A $\beta$ or APMA induced a significant increase in FITC-dextran flux (2.9- to 3.3-fold change) at $24 \mathrm{~h}$ compared with the control, while inhibition of MMP-9 activity by GM6001 or by MMP-9 siRNA partially abolished $\mathrm{A} \beta$-induced high permeability. The figure shows a representative experiment repeated three times with similar results $\left({ }^{* *} \mathrm{P}<0.01\right.$ and $\left.{ }^{*} \mathrm{P}<0.05\right)$.

hypothesis, haRPE cells were treated with $\mathrm{OA} \beta(0.3 \mu \mathrm{M})$ or with APMA $(100 \mu \mathrm{M})$ to induce MMP activation, and the barrier structure was evaluated by immunostaining of TJ proteins. The barrier function was evaluated by measuring TER and passive permeation of FITC-dextran. In the haRPE monolayers cultured with DMEM/F12 as control (Fig. 4A), no morphological change was observed; actin filaments were regular with no breaks in the staining pattern, and the distribution of occludin and $\mathrm{ZO}-1$ was continuous and regular around the cells. Exposure to $\mathrm{A} \beta$ (Fig. 4B) resulted in an irregular morphology and a disturbed distribution of F-actin, ZO-1 and occludin; the staining of F-actin was interrupted in the intercellular space. The staining of occludin showed a diffuse cytoplasmic distribution, and the abnormal distribution of ZO-1 was typically manifested as fragmental staining. The alterations caused by $A \beta$ (Fig. 4B) suggest that $A \beta$ may be associated with RPE dysfunction in AMD. Notably, the deleterious effects of $A \beta$ were reproduced by stimulation with APMA (Fig. 4C) which is known as an MMP agonist. Further study was performed to examine whether this abnormality was associated with a compromised barrier function following exposure to A $\beta$ or APMA. The TER (Fig. 5A) was recorded to determine the stability of TJs, and the transepithelial diffusion rate of FITC-dextran (Fig. 5B) was measured to evaluate the permeability of the monolayers. The result revealed that the TER increased rapidly during the initial 12 days of standard culture and reached a plateau within the following 3 days (Fig. 5A). A mean level of $138 \pm 8.5 \Omega \times \mathrm{cm}^{2}$ TER was recorded on day 15 , and then the monolayer was stimulated as previously described and the TER was measured every day. We observed that exposure of haRPE cells to A $\beta$ or APMA gradually decreased the TER (Fig. 5A) and increased the diffusion rate of FITCdextran (Fig. 5B). These results suggest that activation of MMP-9 by A $\beta$ or APMA caused disruption of TJ proteins and transepithelial permeability dysfunction.
siRNA targeting MMP-9 and GM6001 prevents $A \beta$-induced disruption of barrier integrity. To confirm whether MMP-9 is responsible for disruption of epithelial barrier integrity, haRPE cells were transfected with $40 \mathrm{nM}$ MMP-9 siRNA or were pretreated with GM6001 $(20 \mu \mathrm{g} / \mathrm{ml})$ to inhibit MMP activation prior to $A \beta$ stimulation. Pretreatment with GM6001 inhibited the deleterious effects of $A \beta$ on TJ proteins in the RPE cells (Fig. 4D), and partially attenuated the decrease in TER (Fig. 5A) and the increase in permeability resulting from $A \beta$ treatment (Fig. 5B). These results suggest that inhibition of MMP activation may reverse the deleterious effects of A $\beta$. Exposure of MMP-9-silenced haRPE cells to $\mathrm{A} \beta$ did not induce abnormal staining of TJ proteins (Fig. 4E). Furthermore, transfection with MMP-9 siRNA in haRPE cells attenuated $\mathrm{A} \beta$-induced transepithelial permeability dysfunction (Fig. 5). We demonstrated for the first time that inhibition of MMP-9 activity using RNA interference strategy attenuated $\mathrm{A} \beta$-induced disruption of $\mathrm{TJ}$ proteins and permeability dysfunction.

\section{Discussion}

The BRB plays an important role in the homeostatic regulation of the microenvironment in the retina. Disruption of oBRB is associated with development of AMD. Our study showed for the first time that A $\beta$-induced secretion of MMP-9 is associated with disruption of barrier integrity in primary human adult RPE cells. Stimulation with A $\beta$ promoted release of pro-MMP-9 and active-MMP-9 from haRPE cells (Fig. 2). Exposure of haRPE cells to OA $\beta$ resulted in a disruption of F-actin, ZO-1 and occludin (Fig. 4), loss of TER (Fig. 5A) and increased permeability (Fig. 5B). The results suggest that $A \beta$ may be associated with the reduced barrier function of RPE cells in AMD. We then compared the morphological change (Fig. 4) and barrier functional analysis of monolayers (Fig. 5) in the APMA- and A $\beta$-stimulated group, and 


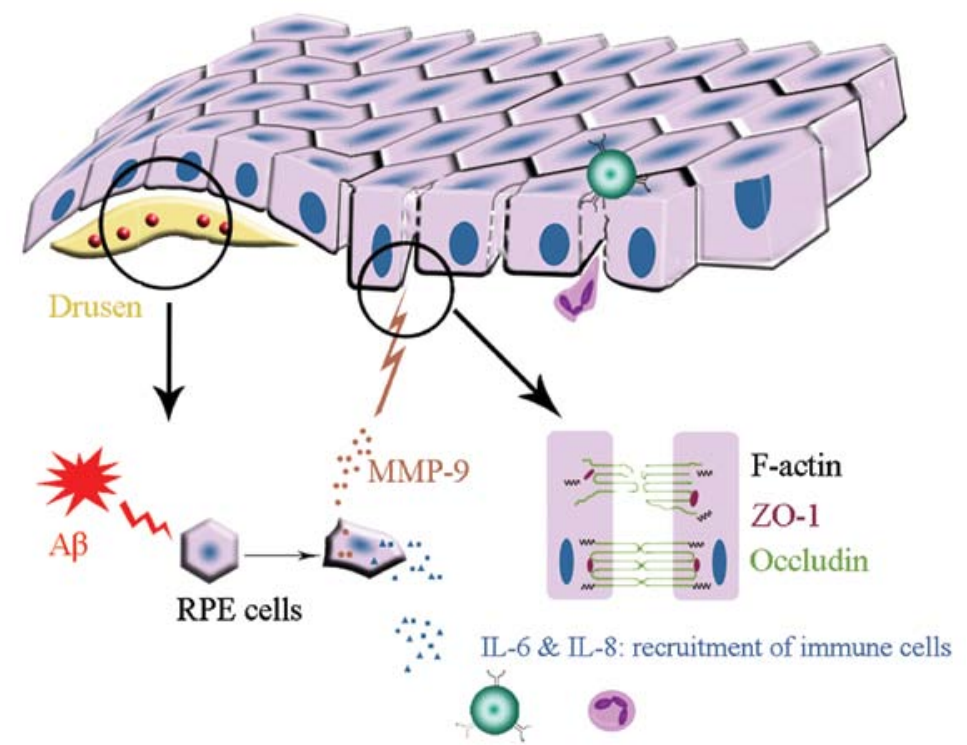

Figure 6. MMP-9 secreted by A $\beta$-stimulated cells disrupts the RPE cell barrier structure and contributes to chronic inflammation. A $\beta$ stimulation induces RPE cells to secrete MMP-9 and proinflammatory cytokines such as IL-6 and IL-8. MMP-9 contributes to oBRB breakdown via proteolysis of epithelial tight junction proteins. IL- 6 and IL-8 are required for recruitment of immune cells. All these factors contribute to continuous chronic inflammation in the retina. RPE, retinal pigment epithelial; ZO-1, zonula occludens-1; oBRB, outer blood-retinal barrier.

similar effects were observed. This suggests that activation of MMP is associated with A $\beta$-induced barrier disruption. Furthermore, inhibition of MMP-9 activity by GM6001 or by MMP-9 siRNA abolished the deleterious effects of $\mathrm{A} \beta$ on TJ ptoreins (Fig. 4) and barrier function of RPE cell monolayers (Fig. 5).

MMPs are calcium-requiring, zinc-containing endopeptidases which constitute a major component of the enzyme cascade responsible for degradation of extracellular matrix proteins such as collagen, proteoglycan and laminin. MMPs can be secretory or cell surface bound. Under normal conditions, MMP activity is required for tissue remodeling, but altered MMP activity has been observed in diseases. Higher concentration of MMP-9 was measured in aqueous humour (22) and plasma (29) of AMD patients. The vitreous MMP-9 level was positively correlated with the severity of AMD (23). A recent study on genome-wide association scan (GWAS) identified AMD susceptibility loci near TIMP3, a metalloproteinase involved in degradation of the extracellular matrix and implicated in early-onset AMD (30).

The TJ proteins of RPE cells are dynamic structures with a variable permeability depending on local factors secreted by RPE cells (31). Accumulated evidence suggests that MMPs may induce barrier breakdown by regulating the structures of TJ proteins. Knockdown of MMP-2 and MMP-9 in leukemic cells reversed the disruption of TJ proteins (19). Activation of MMP-9 disrupted corneal epithelial barrier function by proteolytic cleavage of occludin (26). Although the level of MMP-9 is correlated with the development of AMD, it is unclear what controls MMP secretion from RPE cells and subsequent activation. Complement activation resulted in a loss of TER and accompanying elevated expression of MMP-2 and MMP-9 in RPE cells (32). A recent study demonstrated that $A \beta$-induced increase in MMP secretion from endothelial cells contributed to disruption of ZO-1 expres- sion (33), but the role of MMP-9 in regulating RPE barrier integrity is largely unknown. The present study demonstrated for the first time that $O A \beta$ caused a significant increase in secretion of MMP-9 from RPE cells (Figs. 2 and 3), which was associated with $A \beta$-induced disruption of barrier integrity (Figs. 4 and 5). Recent evidence suggests that $\mathrm{Ca}^{2+}$ deregulation may mediate the cytotoxicity of $A \beta$ on cellular function and viability (33-35). Actually, $A \beta$ in glia can directly increase $\mathrm{Ca}^{2+}$ signals (36). The MMPs constitute a family of proteolytic enzymes that require the binding of $\mathrm{Zn}^{2+}$ and $\mathrm{Ca}^{2+}$ for their enzymatic activity. Therefore, $A \beta$-induced deregulation of $\mathrm{Ca}^{2+}$ homeostasis may explain excessive activation of MMP-9 in cells (37).

$A \beta$, detected in the drusen of AMD, may trigger inflammatory responses in the RPE/choroidal layers of the eye. A $\beta$-induced expression of IL-6, IL-8 and MMP-9 by RPE cells may cause continuous chronic inflammation in AMD for the following reasons (Fig. 6). i) The retina is an immuneprivileged site where inflammatory responses are suppressed, but opening of epithelial barriers by MMP activity may be a mechanism that allows passage of plasma proteins and inflammatory cells into this privileged compartment. ii) The aqueous humour of AMD patients contain higher concentrations of IL-6 and IL-8 (22), which play pivotal roles in leukocyte recruitment. iii) Continued presence (sometimes over many years) of pro-inflammatory factors and immune cells in the retina may cause chronic inflammation. iv) Cytokines secreted by RPE cells may accelerate inflammatory response and cell damage. Together with these observations, our present study suggests that $A \beta$-induced release of MMP-9 contributes to epithelial barrier disruption. Maintenance of the structural integrity in RPE cell monolayers by blocking the action of $A \beta$ or $A \beta$-mediated MMP-9 secretion may thus represent a new approach to the treatment of AMD. 


\section{Acknowledgements}

This study was supported by the Science and Technology Commission of Shanghai (11JC1409900). We gratefully thank the Biochemistry and Molecular Biology Institute of Shanghai Tenth People's Hospital for the technological support, the Xiaoqing Liu's Laboratory of Tongji University for the experimental facilities and equipment and Xiujuan Shi for the excellent technical assistance.

\section{References}

1. Klein R, Peto T, Bird A and Vannewkirk MR: The epidemiology of age-related macular degeneration. Am J Ophthalmol 137: 486-495, 2004

2. Johnson LV, Forest DL, Banna CD, et al: Cell culture model that mimics drusen formation and triggers complement activation associated with age-related macular degeneration. Proc Natl Acad Sci USA 108: 18277-18282, 2011

3. Luibl V, Isas JM, Kayed R, Glabe CG, Langen R and Chen J: Drusen deposits associated with aging and age-related macular degeneration contain nonfibrillar amyloid oligomers. J Clin Invest 116: 378-385, 2006.

4. Mullins RF, Russell SR, Anderson DH and Hageman GS Drusen associated with aging and age-related macular degeneration contain proteins common to extracellular deposits associated with atherosclerosis, elastosis, amyloidosis, and dense deposit disease. FASEB J 14: 835-846, 2000.

5. Isas JM, Luibl V, Johnson LV, et al: Soluble and mature amyloid fibrils in drusen deposits. Invest Ophthalmol Vis Sci 51: 1304-1310, 2010

6. Wang J, Ohno-Matsui K, Yoshida T, et al: Amyloid-beta up-regulates complement factor $\mathrm{B}$ in retinal pigment epithelial cells through cytokines released from recruited macrophages/microglia: another mechanism of complement activation in age-related macular degeneration. J Cell Physiol 220: 119-128, 2009.

7. Wang J, Ohno-Matsui K, Yoshida T, et al: Altered function of factor I caused by amyloid beta: implication for pathogenesis of age-related macular degeneration from drusen. J Immunol 181: 712-720, 2008.

8. Kurji KH, Cui JZ, Lin T, et al: Microarray analysis identifies changes in inflammatory gene expression in response to amyloid-beta stimulation of cultured human retinal pigment epithelial cells. Invest Ophthalmol Vis Sci 51: 1151-1163, 2010.

9. Streilein JW: Immune privilege as the result of local tissue barriers and immunosuppressive microenvironments. Curr Opin Immunol 5: 428-432, 1993

10. Jo DH, Kim JH and Kim JH: How to overcome retinal neuropathy: the fight against angiogenesis-related blindness. Arch Pharm Res 33: 1557-1565, 2010.

11. Cunha-Vaz J, Bernardes R and Lobo C: Blood-retinal barrier. Eur J Ophthalmol 21: 3-9, 2010.

12. Bailey TA, Kanuga N, Romero IA, Greenwood J, Luthert PJ and Cheetham ME: Oxidative stress affects the junctional integrity of retinal pigment epithelial cells. Invest Ophthalmol Vis Sci 45 675-684, 2004

13. Peng S, Gan G, Rao VS, Adelman RA and Rizzolo LJ: Effects of proinflammatory cytokines on the claudin-19 rich tight junctions of human retinal pigment epithelium. Invest Ophthalmol Vis Sci 53: 5016-5028, 2012.

14. Liu J, Jin X, Liu KJ and Liu W: Matrix metalloproteinase-2-mediated occludin degradation and caveolin-1-mediated claudin-5 redistribution contribute to blood-brain barrier damage in early ischemic stroke stage. J Neurosci 32: 3044-3057, 2012.

15. Martins T, Baptista S, Gonçalves J, et al: Methamphetamine transiently increases the blood-brain barrier permeability in the hippocampus: role of tight junction proteins and matrix metalloproteinase-9. Brain Res 1411: 28-40, 2011.

16. Bruban J, Glotin AL, Dinet V, et al: Amyloid-beta(1-42) alters structure and function of retinal pigmented epithelial cells Aging Cell 8: 162-177, 2009.

17. Maminishkis A, Chen S, Jalickee $S$, et al: Confluent monolayers of cultured human fetal retinal pigment epithelium exhibit morphology and physiology of native tissue. Invest Ophthalmol Vis Sci 47: 3612-3624, 2006
18. Suzuki T, Yoshinaga N and Tanabe S: Interleukin-6 (IL-6) regulates claudin-2 expression and tight junction permeability in intestinal epithelium. J Biol Chem 286: 31263-31271, 2011.

19. Feng S, Cen J, Huang Y, et al: Matrix metalloproteinase-2 and -9 secreted by leukemic cells increase the permeability of bloodbrain barrier by disrupting tight junction proteins. PLoS One 6: e20599, 2011.

20. Bauer AT, Bürgers HF, Rabie T and Marti HH: Matrix metalloproteinase-9 mediates hypoxia-induced vascular leakage in the brain via tight junction rearrangement. J Cereb Blood Flow Metab 30: 837-848, 2010.

21. Manicone AM and McGuire JK: Matrix metalloproteinases as modulators of inflammation. Semin Cell Dev Biol 19: 34-41, 2008.

22. Jonas JB, Tao Y, Neumaier M and Findeisen P: Cytokine concentration in aqueous humour of eyes with exudative agerelated macular degeneration. Acta Ophthalmol 90: e381-e388, 2012.

23. Ecker SM, Pfahler SM, Hines JC, Lovelace AS and Glaser BM: Sequential in-office vitreous aspirates demonstrate vitreous matrix metalloproteinase 9 levels correlate with the amount of subretinal fluid in eyes with wet age-related macular degeneration. Mol Vis 18: 1658-1667, 2012.

24. Jeong S, Ledee DR, Gordon GM, et al: Interaction of clusterin and matrix metalloproteinase-9 and its implication for epithelial homeostasis and inflammation. Am J Pathol 180: 2028-2039, 2012.

25. Huet E, Vallée B, Delbé J, et al: EMMPRIN modulates epithelial barrier function through a MMP-mediated occludin cleavage: implications in dry eye disease. Am J Pathol 179: 1278-1286, 2011.

26. Pflugfelder SC, Farley W, Luo L, et al: Matrix metalloproteinase-9 knockout confers resistance to corneal epithelial barrier disruption in experimental dry eye. Am J Pathol 166: 61-71, 2005

27. Kayed R, Head E, Thompson JL, et al: Common structure of soluble amyloid oligomers implies common mechanism of pathogenesis. Science 300: 486-489, 2003.

28. Giebel SJ, Menicucci G, McGuire PG and Das A: Matrix metalloproteinases in early diabetic retinopathy and their role in alteration of the blood-retinal barrier. Lab Invest 85: 597-607, 2005.

29. Chau KY, Sivaprasad S, Patel N, Donaldson TA, Luthert PJ and Chong NV: Plasma levels of matrix metalloproteinase-2 and -9 (MMP-2 and MMP-9) in age-related macular degeneration. Eye 22: 855-859, 2008

30. Chen W, Stambolian D, Edwards AO, et al: Genetic variants near TIMP3 and high-density lipoprotein-associated loci influence susceptibility to age-related macular degeneration. Proc Natl Acad Sci USA 107: 7401-7406, 2010.

31. Balda MS, Gonzalez-Mariscal L, Contreras RG, et al: Assembly and sealing of tight junctions: possible participation of G-proteins, phospholipase C, protein kinase $\mathrm{C}$ and calmodulin. J Membr Biol 122: 193-202, 1991.

32. Bandyopadhyay $M$ and Rohrer B: Matrix metalloproteinase activity creates pro-angiogenic environment in primary human retinal pigment epithelial cells exposed to complement. Invest Ophthalmol Vis Sci 53: 1953-1961, 2012.

33. Kook SY, Hong HS, Moon M, Ha CM, Chang S and Mook-Jung I: $\mathrm{A} \beta_{1-42}$-RAGE interaction disrupts tight junctions of the bloodbrain barrier via $\mathrm{Ca}^{2+}$-calcineurin signaling. J Neurosci 32: 8845-8854, 2012.

34. Thibault O, Gant JC and Landfield PW: Expansion of the calcium hypothesis of brain aging and Alzheimer's disease: minding the store. Aging Cell 6: 307-317, 2007.

35. Grolla AA, Fakhfouri G, Balzaretti G, et al: A $\beta$ leads to $\mathrm{Ca}^{2+}$ signaling alterations and transcriptional changes in glial cells. Neurobiol Aging 34: 511-522, 2013.

36. Kuchibhotla KV, Lattarulo CR, Hyman BT and Bacskai BJ: Synchronous hyperactivity and intercellular calcium waves in astrocytes in Alzheimer mice. Science 323: 1211-1215, 2009.

37. Kobayashi T, Hattori S, Nagai Y and Tajima S: Differential regulation of the secretions of matrix metalloproteinase-9 and tissue inhibitor of metalloproteinases-1 from human keratinocytes in culture. IUBMB Life 50: 221-226, 2000. 